\title{
Targeting melanoma cells with a drug-infused graphene matrix
}

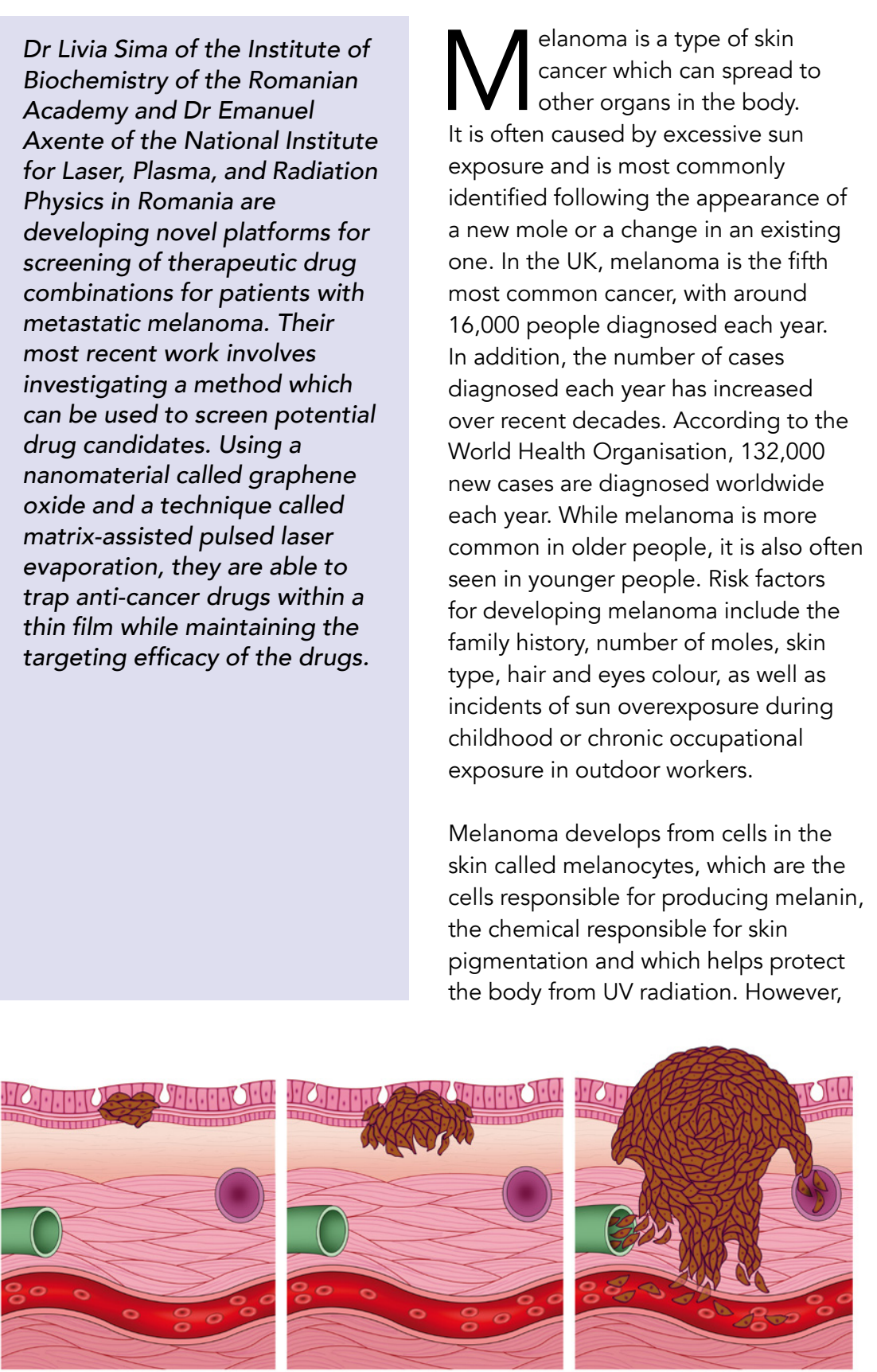

Metastatic melanoma can pass into blood vessels, facilitating the spread to other parts of the body. too much exposure to the sun's rays can damage the genetic material of skin cells (heir DNA). This damage can lead to uncontrolled proliferation of cells, Ulimately resulting in cancer. Metastatic originated in the skin spreads to other tissues of the body Cells spread from the original tumour into surrounding tissue, and from here they are able to pass into blood vessels. The blood vessels are then able to transport the cancer cells around the body. facilitating the spread of disease.

There are a range of different treatment options for melanoma, including surgery, radiotherapy and chemotherapy. However, in some cases, chemotherapy can lead to the development of resistance, where the cancer cells evolve to avoid the letha Thects of the chemotherapy drugs. Therefore, there is an urgent need that can be used as part of the cancer treant toolkt Doctors now tend to

se a personalised approach to treating cancer, rather than a blanket 'one size fits all' approach. This means tha several treatment options should be available for the patient and they can be used in the most effective way to prevent the progression of melanoma.

The prognosis for patients with metastatic melanoma used to be poor with limited therapeutic options and an average prognosis of 6-9 months to live after diagnosis. In 2011, there was a major breakthrough in melan treatment. a new immunotherapy the researchers a Nobel Prize. When

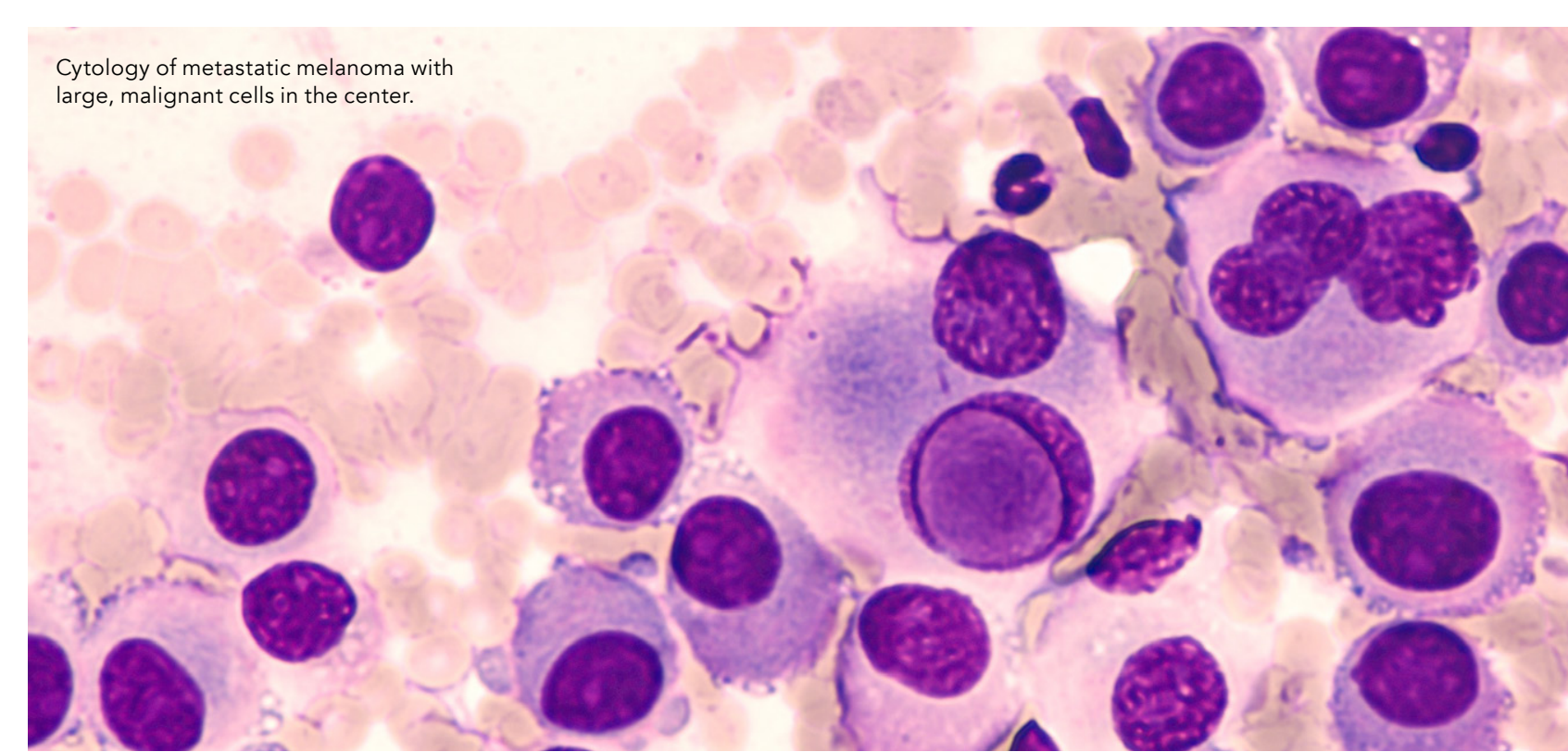

injected, this treatment released the brakes of the body's immune cells and cells. However, not all patients respona to this treatment, so alternative treatments are still required.

NOVEL CANCER TREATMENTS Dr Livia Sima of the Institute of Biochemistry of the Romanian Academy and Dr Emanuel Axente of the National Institute for Laser, Plasma, and Radiation Physics in Romania are developing testing platforms for novel therapeutic drug combinations for patients with metastatic melanoma.

Some patients with melanoma have particular mutations in a gene called PRAF, a proten which is usually involved rowth In some cancers this gene is mutated, resulting in defective cell

In the UK, melanoma is the fifth most common cancer, with around 16,000 people diagnosed each year, while 132,000 new cases are reported worldwide each year.

division. One particular mutation of the to an increasing number of cells which BRAF gene, V6O0E, is present in around are able to survive treatment with these $60 \%$ of melanoma-related tumours.

There are already drugs available which target cancers driven by mutations in the BRAF gene, including vemurafenib and dabratenib. These drugs, called BRAF inhibitors, usually act by interfering with the action of BRAF, thus intemupting upregulation of cell proliferation.

Nevertheless, some cancer cells escape .

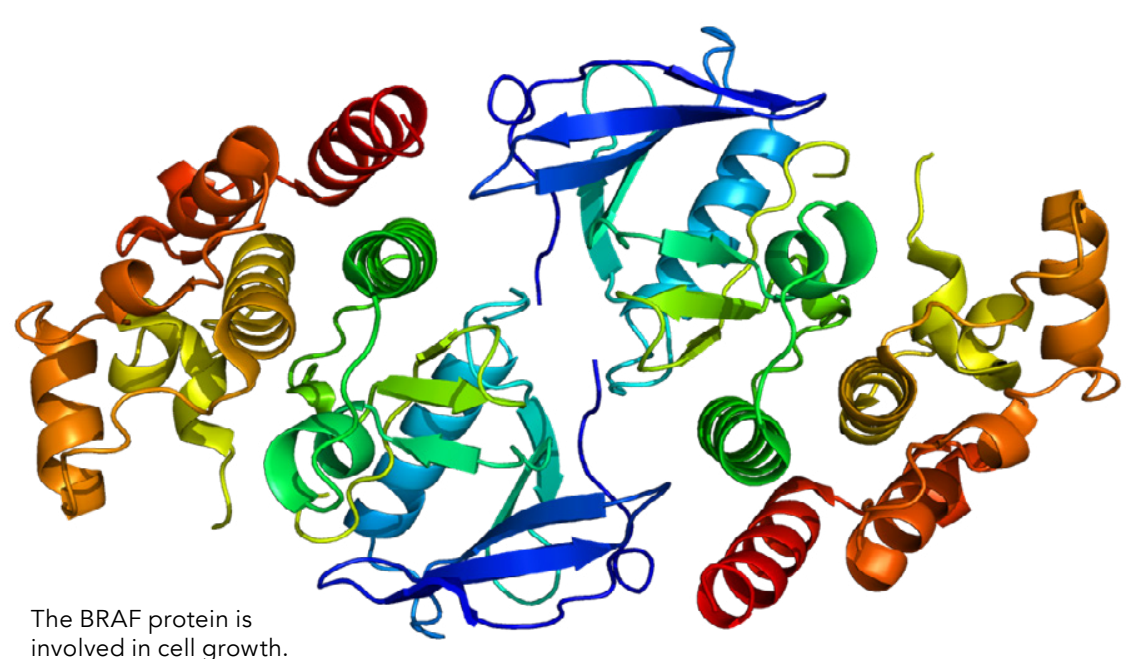
which are resistant to treatment.

There are ways in which these resistan cells can be re-sensitised to BRAF inhibitors. Recent studies showed that epigenetic modulators, such as proteins called histone deacetylase proliferation of can, could help stop the prolferation of cancer cells. What's th expression of other protein molecules, some of which help cancer cells evade drugs and host immune cells. By inhibiting the action of HDACs, it is possible to take the brakes of this immune suppressive effect, as well as re-sensitise cancer cells to BRAF inhibitors. The research of Dr Sima and DrAxente aims to harness existing anti-melanoma drugs and use them to test new techniques for screening future candidate drugs.

EXPLOITING CANCER'S ADDICTION TO DRUGS Cancer cells can become addicted So the drugs designed to treat them. 


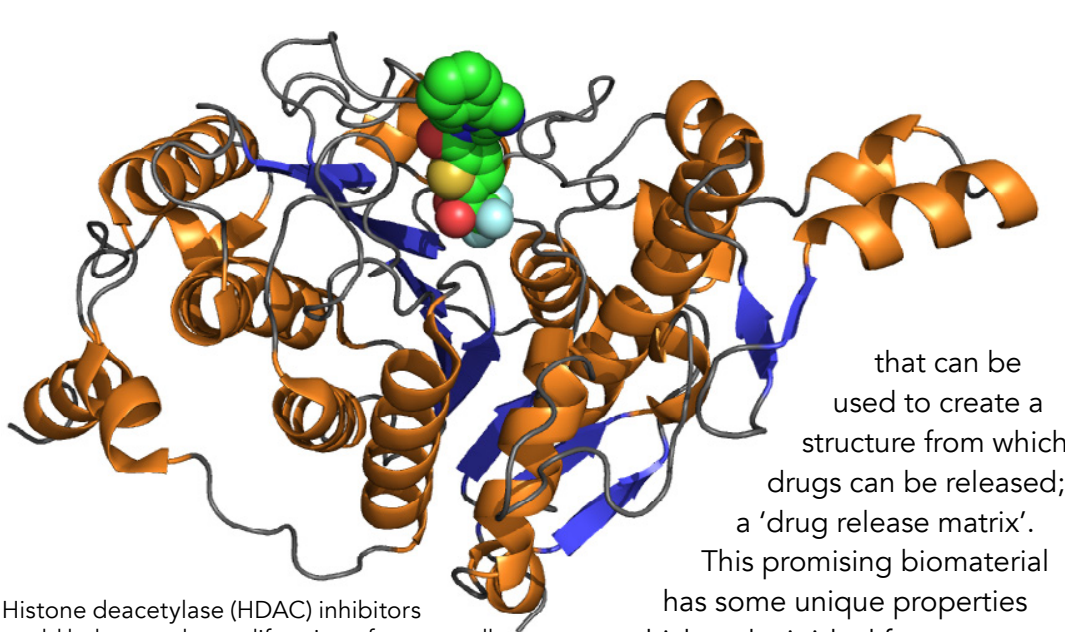

ich make it ique properties

a drug delivery system, including biocompatibility, a large surface area and chemical stability.

symptoms when particular treatments e stopped, including slowed grow

Dr Sima and Dr Axente aim to build Dinatorial platforms that can combinations using cells outc drug patients. There is a need for highly specialised techniques to explore the elationships between cancer cells and iomaterials such as graphene oxid GO), which can be used for drug delivery, so that novel treatments for cancer can be developed. The first challenge the research team had to overcome was how to get the drug to the target cells. They used GO as a vehicle to deliver either BRAF as a vitors or HDAC inh melanoma cells.

\section{THE MAGIC CARPET OF}

The researchers validated the functionality of the drug-infused deposition techniques. Matrix-assisted pulsed laser evaporation (MAPLE) is

Dr Sima and Dr Axente created a thin coating comprised of graphene oxide that incorporated the existing drugs dabrafenib or trichostatin $\mathrm{A}$. These drugs are designed to inhibit the action of cells expressing the V 600 mutation in their BRAF gene, one of the most prevalent modifications encountered in melanoma tumours or that of HDACs epigenetic enzymes, respectively. In doing so, the researchers were able to show that cancer cells exposed to this drug-containing graphene coating decreased in activity. The results showed that the graphene matrices se, and that the released drugs stil produced dose-dependent molecular effects on human melanoma cell lines.

Drug activity was maintained, even when the drugs were incorporated into a graphene oxide matrix and transferred by laser on a testing platform.

a new technique which can be used to fabricate uniform, thin layers of nanomaterials, such as graphene oxide. It is based on the concept of pulsed laser deposition, where high-power laser beam is aimed at It is similar to the co be deposted. bs which contained anti-cancer drugs
Graphene oxide nanomaterials were successfully immobilised on a solid underlying plattorm using MAPLE techrique. Drug activity was maintained, even when the drugs were matrix and transf gaphe oxide by a laser beam. This type of function platform has potential to screen other candidate drugs. If drugs can safely be immobilised in a nanomaterial and the cancer cells then exposed to this druginfused matrix, this offers an effective, high-throughput method to test the effectiveness of new anti-cancer drugs and combinations thereof.

Future work of Dr Sima and Dr Axente will involve testing the synergistic effects of anti-cancer compounds using novel techniques such as combinatorial MAPLE, as well as microfluidic 3D models designed intercellular cross-talk found in hum patients' tumours.

\section{Behind the Research}

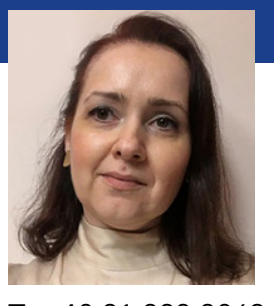

Dr Livia

Sima

E: Isima@biochim.ro

(1)

com/in/livia-sima-3abob116

Research Objectives

Dr Livia Sima and Dr Emanuel Axente are developing platforms for screening of therapeutic drug combinations for patients with metastatic melanoma.

\section{Detail}

Livia Sima

Institute of Biochemistry of the

Romanian Academy (IBAR)

296 Splaiul Independente

Romania

Emanuel Axente

Photonic Investigations Laboratory

Center for Advanced Laser

National Institute for Laser, Plasma

409 Atomistilor Stree RO-77125 Magurele
Romania

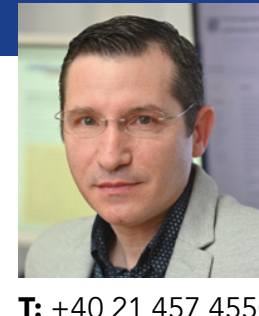

Dr Emanuel

Axente

E: emanuel.axente@inflpr.ro

T: +40 214574550 W: http://cetal.inflpr.ro/newsite/ W: http://cetal.inflpr.ro/newsi

Bio

Dr Livia Sima completed her PhD at the Institute of Biochemistry of the postdoctoral studies at Northweste University, USA. Upon completion of her training, she returned to IBAR
where she occupies a Senior Scientific where she occupies a Senior Scientific Researcher II position working as TG2TARGET projects.

Dr Emanuel Axente is Senior Scientific Researcher I at the National Institute for Laser, Plasma and

Radiation Physics, 1 his $\mathrm{PhD}$ in physics from University of Bucharest in coDrance. He is the ead of Photonic Investigations

Romanian Ministry of Education and Research

Collaborators

- Dr Felix Sima, Photonic Investigations Laboratory, CETAL, INFLPR, Romania Dr Gabriela Chiritoiu, Molecular Romania

\section{References}

Sima, L.E., Chiritoiu, G., Negut, I., Grumezescu, V., Orobeti, S. Muntea, G, NVVA, Sima, F. and Axente, E. (2020). Functionalized Graphene Oxide Thin Films for Anti-tumor Drug Delivery to Melanoma Cells. Frontiers in Chemistry, 8(184). Available at: https://www.doi. org/10.3389/fchem.2020.00184

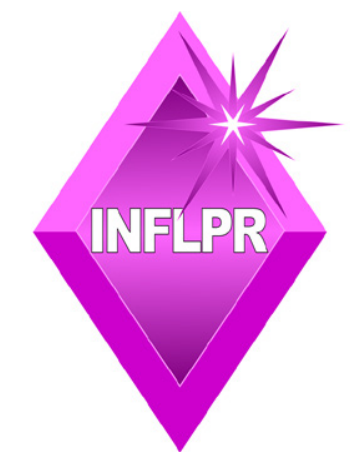

\section{Personal Response}

In what other ways do you envisage graphene oxide being used for drug development and testing?

II Personalised cancer therapeutic strategies could be achieved by designing new drug combinations. Developing innovative testing plattorms will allow screening the combinations for synergistic targeting be easily functionalised with a broad spectrum of molecules in view of fabricating 2D compositional mixtures. Moreover, drug-loaded graphene oxide nano-carriers could be good candidates to be tested in $3 \mathrm{D}$ microfluidic set-ups fabricated to mimic tumour
microenvironment.

Institute of Biochemistry 